\title{
OPTIMALITY CONDITIONS FOR NONSMOOTH OPTIMISATION PROBLEMS VIA GENERALISED DERIVATIVES
}

\author{
SARA HASSANI \\ (Received 5 October 2017; first published online 4 December 2017)
}

2010 Mathematics subject classification: primary 49M05; secondary 90C30.

Keywords and phrases: optimal control, generalised derivative, topical function, infinite horizon optimisation.

The motivation for generalising the concept of subdifferentials is inspired from many real world applications involving nonsmooth functions. These generalisations of the derivative have been used in many applications including optimal control, mathematical programming and constrained optimisation. They are also very important tools to derive optimality conditions.

In this thesis, first, we consider optimality conditions for optimisation problems by introducing new notions of supporting cones, including local and global supporting cones [2, 6, 7]. In the development of these concepts, we use the notion of the augmented normal cone that has been frequently considered in the literature. These introduced concepts of supporting cones together with the so-called weak subdifferentials are used to derive optimality conditions for local and global optima $[3,4]$. Then, the results obtained are extended to reflexive Banach spaces [8].

Next, optimisation problems for topical functions are investigated. They arise in a remarkable variety of mathematical disciplines such as matrices over the maxplus semiring, Leontief substitution systems of mathematical economics, dynamic programming operators of games and of Markov decision processes and nonlinear operators arising in matrix scaling problems and demographic modelling. We first give characterisations of the superdifferential of extended valued topical functions defined on a semimodule with values in a semifield. Next, as an application, we present necessary and sufficient conditions for global maxima of the difference of two strictly topical functions defined on a semimodule by using the superdifferential of extended valued topical functions [9].

Thesis submitted to Federation University in October 2016; degree approved on 15 June 2017; supervisors Musa Mammadov and Alex Kruger.

(c) 2017 Australian Mathematical Publishing Association Inc. 0004-9727/2017 \$16.00 
Finally, we devote the last section to infinite horizon optimisation problems, which have many applications in real world problems; for example, in capacity expansion, equipment replacement and production planning. The objective of these problems is to find a sequence of decisions such that the associated cost over an unbounded horizon is optimal. In this thesis, we investigate the mathematical formulation for horizon optimisation that has been commonly studied in the literature. This involves the description of a mathematical model for a sequence of decisions and associated costs, as well as the metric in the space of infinite decisions that are used to study the convergence of the minimising sequence. We also investigate the convergence of a sequence of trajectories under the assumption that the functional values (total costs) converge to the optimal value (that is, the minimal total cost). The convergence is considered in the sense of ideals and the particular case is called statistical convergence [5].

Moreover, we investigate the optimality condition for the minimum cost of infinite horizon optimisation by introducing two new notions, contingent cone and upper contingent derivative. The notion of contingent cone to the set of trajectories in infinite horizon optimisation problems is introduced. Some important properties of contingent cones are investigated. Also the notion of upper contingent derivative is introduced, based on the same idea used in the literature when defining directional derivatives. Then, optimality conditions are derived in terms of the contingent cone and the upper contingent derivative [1].

\section{References}

[1] S. Hassani and M. Mammadov, 'Optimality conditions in infinite horizon optimization by contingent derivative', Pac. J. Optim., to appear.

[2] S. Hassani and M. Mammadov, 'Relation between weak subdifferentials and Hadamard lower directional derivative', in Proc. 44th Iranian Mathematics Conference, Mashhad, Iran, 2013, (Iranian Mathematical Society, Teheran, Iran, 2013), 348-351.

[3] S. Hassani and M. Mammadov, 'Necessary and sufficient conditions for local optimality via weak subdifferentials', Adv. Theor. Appl. Math. 9(2) (2014), 143-154.

[4] S. Hassani and M. Mammadov, 'Sigma supporting cone and optimality conditions in non-convex problems', Far East J. Math. Sci. (FJMS) 91 (2014), 169-190.

[5] S. Hassani and M. Mammadov, 'Convergence of trajectories in infinite horizon optimization', Int. J. Nonlinear Anal. Appl. 8(1) (2017), 123-131.

[6] S. Hassani, M. Mammadov and M. Jamshidi, 'Characterizing non-convex sets with conic gap via sigma supporting cone', in Proc. 10th IMT-GT International Conference on Mathematics, Statistics and its Applications, Terengganu, Malaysia, 2014, (American Institute of Physics, New York, 2014), 143-148.

[7] S. Hassani, M. Mammadov and M. Jamshidi, 'Some geometrical properties of non-convex sets in finite dimensions', in Proc. 45th Iranian Mathematics Conference, Semnan, Iran, 2014, (Iranian Mathematical Society, Teheran, Iran, 2014), 112-114.

[8] S. Hassani, M. Mammadov and M. Jamshidi, 'Optimality conditions via weak subdifferentials in reflexive Banach spaces', Turkish J. Math. 41 (2017), 1-8.

[9] S. Hassani and H. Mohebi, 'Characterizations of minimal elements of topical functions on semimodules with applications', Linear Algebra Appl. 520 (Suppl. C) (2017), 104-124. 
SARA HASSANI, School of Physical, Environmental and Mathematical Sciences, University of New South Wales, Canberra, Australia e-mail: s.hassani@unsw.edu.au 